\title{
Guía de redacción: reporte de un caso
}

\author{
Writing guide: a case report
}

\section{José María Jiménez Ávila, * Lorena Alejandra García Rosas *}

*Instituto Tecnológico y de Estudios Superiores de Monterrey, Campus Guadalajara. Guadalajara, Jalisco, México. Centro Médico Nacional de Occidente. Instituto Mexicano del Seguro Social. Unidad Médica de Alta Especialidad. Clínica de Columna.

\begin{abstract}
Resumen
El reporte de caso es un tipo de publicación de fuente primaria que permite aprender a identificar el comportamiento de una patología que tiene una característica especial. Aunque tiene un nivel bajo de evidencia (IV), éste puede favorecer la comprensión de las guías de práctica clínica, la forma cómo se da el razonamiento clínico y el manejo de la incertidumbre. El objetivo es contribuir con aspectos nuevos de una enfermedad determinada, donde se toma en cuenta la novedad, originalidad e interés del caso que se presenta, es decir, una descripción no descrita previamente, poco frecuente o rara se puede considerar una evolución inusual de la sintomatología de la enfermedad. Su estructura debe basarse en la presentación de los síntomas del caso, una descripción detallada de las pruebas y exámenes, el diagnóstico, la descripción del tratamiento prescrito y los resultados obtenidos, acompañados de una discusión y conclusión del caso y sustentando con la revisión de la literatura previamente citada.
\end{abstract}

Palabras clave: Manuscrito, publicación, caso, evidencia.

\section{Abstract}

The case report is a type of primary source publication, which allows learning to identify the behavior of a pathology that has a specific characteristic; although it has a low level of evidence, this can favor the understanding of the practice guidelines clinical, how clinical reasoning and the handling of uncertainty occur. The objective is to contribute with new aspects of a given disease, where the novelty, originality, and interest of the case presented are taken into account. This is a description not previously reported, infrequent or rare; it can be considered an unusual evolution of the symptomatology of the disease. Its structure should depend on the presentation of the symptoms of the case, a detailed description of tests and examinations, diagnosis, description of the prescribed treatment, and the results obtained, accompanied by a discussion and conclusion of the case and supported by the review of the previously cited literature.

Keywords: Manuscript, publication, case, evidence.

\section{Introducción}

Las revistas de las ciencias de la salud incluyen diferentes tipos de publicaciones científicas, entre ellas el reporte de un caso es una publicación de origen primario y se denomina así porque presenta resultados de una investigación original (no reportados previamente). Es importante recalcar que no se debe confundir el diseño de investigación con el tipo de publicación científica. . $^{1-5}$

Los primeros informes de casos clínicos aparecieron en revistas médicas alrededor del mundo hace más de un siglo como las primeras formas

Correspondencia:

José María Jiménez Ávila

E-mail: josemajimeneza@hotmail.com de comunicación entre clínicos; se consideran el primer peldaño en la investigación clínica, seguido de la serie de casos, el estudio de casos y controles y así sucesivamente. ${ }^{2,6}$

Compartir la experiencia basada en la evidencia y en específico de los casos clínicos facilita el aprendizaje continuo del profesional y permite conocer y actualizar las destrezas médico-quirúrgicas.

Pese a tener un nivel bajo de evidencia científica (nivel IV, Shekelle), ${ }^{4}$ la publicación de un caso clínico favorece la comprensión de una guía de práctica clínica, la forma de razonamiento y el manejo de la incertidumbre; su revisión definitiva-

Recibido: 17-04-2021. Aceptado: 24-04-2021.

Citar como: Jiménez ÁJM, García RLA. Guía de redacción: reporte de un caso. Orthotips. 2021; 17 (3): 152-154. https://dx.doi. org/10.35366/100625 
mente incrementa la gama del diagnóstico diferencial y estimula la mejora continua en el manejo de la patología, que en ocasiones tiene resultados inesperados.

En este tipo de publicación se presenta como un caso clínico con un interés especial; (en inglés se describe como case report o clinical case), el cual puede deberse a su baja frecuencia de aparición, a la presentación clínica poco frecuente de una enfermedad común, o por estar relacionado con aspectos relevantes del diagnóstico o del tratamiento., ${ }^{2,3}$

El caso clínico puede ser publicado en revistas médicas, en congresos científicos y en sesiones clínicas y su discusión permite el intercambio de conocimientos y experiencia, lo que resulta muy útil para el aprendizaje. ${ }^{2}$

La redacción debe seguir principios generales relacionados con la estructura y el estilo, y su objetivo es contribuir al conocimiento médico, presentando aspectos nuevos o instructivos de una enfermedad determinada.

Por lo regular este tipo de reportes cumplen con algunas o varias de las siguientes características:?

1. Están relacionados con una patología nueva o poco frecuente.

2. Son situaciones que no ocurren con frecuencia.

3. Muestran alguna aplicación clínica importante.

4. Los resultados son diferentes a los esperados habitualmente.

5. Existe una aportación diferente de lo que se hace de manera regular.

6. Son ejemplo de un enfoque práctico y novedoso para el diagnóstico o el manejo quirúrgico de una patología.

7. Sirven para enseñar. ${ }^{2-4}$

\section{Para su elaboración}

El autor debe revisar previamente la literatura médica con el fin de investigar si el caso tiene o no algún precedente o si ha sido descrito alguna vez, debe reflejar el razonamiento que se ha seguido a lo largo de todo el proceso diagnóstico y terapéutico, sin olvidar que su redacción debe ser amena para poder cumplir su función docente, y por lo regular se acompaña de la revisión de la literatura, por lo que las palabras clave (keywords) tienen que identificarse claramente para hacer una búsqueda avanzada, crítica y actualizada de los artículos que acompañarán a la revisión. "Todos los médicos tenemos siempre un caso digno de publicar».
Este tipo de publicaciones no debe exceder 1,000 palabras, con un resumen de 200 palabras, tener un mínimo de 20 referencias bibliográficas y el número total de cuadros y figuras por lo general es variable.

El reporte de un caso consta de las siguientes secciones: $2,5,6$

1. Título. Debe ser breve, claro, específico, sencillo, impactante, llamativo, «útil y novedoso».

2. Resumen. Debe ser corto, concreto, fácil de leer. Incluye 200 palabras que describen los aspectos sobresalientes del caso y por qué amerita ser publicado.

3. Introducción. Da una idea específica del tema sustentada con argumentos (epidemiológicos y/o clínicos), la razón por la que se publica, su justificación clínica, o sus implicaciones para la salud pública. Debe realizarse una revisión crítica de la literatura sobre otros casos similares, destacando la gravedad, dificultad para su reconocimiento, forma de presentación y debe incluir un mínimo de 20 artículos como referencias; se sugiere plantear una pregunta para definir las palabras clave y hacer una búsqueda más efectiva.

4. Presentación del caso. Debe describir de manera cronológica los datos de la enfermedad y la evolución del paciente, incluye la sintomatología, la historia clínica pertinente, los datos importantes sobre la exploración física, los resultados de exámenes o pruebas diagnósticas, el tratamiento y el desenlace (mejoría, falta de respuesta o muerte). Debe narrar el proceso para llegar al diagnóstico, y describir de manera precisa las técnicas quirúrgicas o métodos diagnósticos utilizados en el paciente. Se debe proteger la confidencialidad del paciente (omitir el nombre y el número de historia clínica). Si se publica una foto ilustrativa del caso se debe proteger su identidad, describiendo en el pie de figura las características de dicha imagen.

5. Discusión. Es la interpretación de los resultados en el contexto del conocimiento científico prevalente en relación con el mensaje principal y el nuevo conocimiento que aporta este reporte de caso. ${ }^{2}$ Es un recuento de los hallazgos principales del caso clínico, donde se destacan sus particularidades o contrastes, comparándolo con lo ya escrito; se debe sustentar el diagnóstico con evidencia clínica y de laboratorio; se detallan las limitaciones de las evidencias; se debe describir cómo se hizo el diagnóstico diferencial y si otros diagnósticos fueron descartados adecuadamen- 
te. El caso debe compararse con lo ya escrito, sus semejanzas y sus diferencias y se enfatiza lo relevante y cuál es su aportación científica. Es muy importante No hacer generalizaciones basadas en el caso o casos descritos, ya que hay que recordar que el nivel de evidencia es tipo IV. ${ }^{4}$

6. Conclusión. Se resalta alguna aplicación o mensaje claro relacionado con el caso. Se incluyen los comentarios de la solución del caso reseñando sus particularidades científicas, su novedad o cómo se manejó la incertidumbre. Sirve para clarificar aspectos discutibles. Por su finalidad educativa debemos tener una enseñanza que se proyecte en el futuro por medio de recomendaciones para el manejo de pacientes similares o las líneas de investigación que podrían originarse a propósito del caso. $3,4,6$

7. Referencias. Deben ser relevantes, actualizadas y relacionadas con el caso (apegarse al sistema tipo Vancouver y enumerar un mínimo de 20 citas).

8. Anexos. Incluye las figuras y tablas, entendiendo que un caso debe ser lo más gráfico posible y se sugiere que no excedan de siete..$^{2,3,4}$

En el artículo reporte de caso se presentan y describen los resultados de un estudio sobre una situación particular con el fin de dar a conocer las experiencias técnicas y metodológicas y el material obtenido al trabajar con un individuo, organización o situación específica para describir un problema o indicar cómo resolverlo. Esta tipología textual puede estructurarse así:

- $\checkmark$ Título en español e inglés. $\checkmark$ Datos del autor. $\checkmark$ Indicar el tipo de artículo. $\checkmark$ Tabla de contenido numerada. $\checkmark$ Resumen estructurado en español e inglés, 150 palabras.

- $\checkmark$ Introducción: tema, objetivos, tesis, principales fuentes utilizadas. $\checkmark$ Revisión analítica de la literatura sobre casos análogos.

Desarrollo: cuerpo del texto con subtitulación interna.

1. El sujeto y sus características.

2. La naturaleza del problema o su solución mediante la ilustración del ejemplo de caso.

3. Las preguntas que surgen en relación con la investigación o fundamentación teórica adicional.

\section{Lista de referencias}

La estructura de un estudio de caso suele ser la siguiente:

- $\checkmark$ Presentación de los síntomas de un caso concreto.

- $\checkmark$ Descripción detallada de las pruebas y análisis realizados al paciente.

- $\checkmark$ Diagnóstico.

- $\checkmark$ Descripción del tratamiento prescrito.

- $\checkmark$ Resultados obtenidos.

\section{Lista de cotejo}

1. Título

2. Resumen

3. Palabras clave

4. Introducción

5. Presentación del caso

6. Discusión

7. Conclusión

8. Bibliografía

9. Anexos

\section{Referencias}

1. Jeffrey CW. Instructions to authors for submission of articles. Case reports: information for authors. Global Spine Journal. 2012; 1 (1): A1-A6.

2. Villanueva LIS. Cómo redactar un caso clínico. Acta Ortop Mex. 2009; 23 (5): 315-316.

3. Contreras AM, Ochoa-Jiménez RJ. Mensaje principal. En: Manual de redacción científica. Escribir artículos científicos es fácil después de ser difícil: una guía práctica. Guadalajara, Jalisco, México: Ediciones de la Noche; 2010, pp. 53-58.

4. Shekelle P, Woolf S, Eccles M, Grimshaw J. Developing guide lines. BMJ. 1999; 318: 593-596.

5. Matthews JR, Matthews RW. Prepariong to write. En: Successful Scientific Writing. A step-by-step guide for the biological and medical sciencies. $3^{\mathrm{a}}$ ed. New York, USA: Cambridge University Press; 2008, pp. 1-30.

6. Peat J, Elliott E, Baur L, Keena B. Getting Started. In: Peat J, Elliott E, Baur L, Keena B. Scientific writing easy when you know how. London, UK: BMJ Books; 2002.

\section{Conflicto de intereses}

Los autores declaran no tener ningún conflicto de intereses. 